\title{
The safety of elective surgery with concurrent use of immunosuppressants
}

\author{
Katelyn Koons ${ }^{1}$, Victoria Plotas ${ }^{1}$, David S. Tichansky', and Michael R. Kammerer*1 \\ ${ }^{1}$ Division of Minimally Invasive, Metabolic and Bariatric Surgery, Department of Surgery, Thomas Jefferson University, Philadelphia, Pennsylvania, USA
}

\begin{abstract}
While providing superior benefits for a cadre of diverse diseases, the prevalence of Immunosuppressive use often brings to question possible additional risks caused by these therapies in the setting of surgical procedures. Specifically, is the risk of impaired wound healing or increased chance of infection worth avoiding the possible side effects associated with temporary cessation during the perioperative period. The appropriate use of immunosuppressants (IS) during elective surgery has been debated extensively with conflicting results, through the current body of literature. Herein, we aim to provide a comprehensive, yet streamlined, overview of the common IS medications that surgeons are likely to encounter in practice in a wide variety of patients. This discussion includes an overview of the pharmacology and use of eight of the common IS medications: azathioprine, cyclosporine, hydroxychloroquine, infliximab, leflunomide, methotrexate, methylprednisolone, and mercaptopurine.
\end{abstract}

\section{Introduction}

Immunosuppressive therapies have increasingly become the mainstay of treatment for many diseases. While providing superior benefits for a cadre of diverse diseases, the prevalence of their use often brings to question possible additional risks caused by these therapies in the setting of surgical procedures. Specifically, is the risk of impaired wound healing or increased chance of infection worth avoiding the possible side effects associated with temporary cessation during the perioperative period. The appropriate use of immunosuppressants (IS) during elective surgery has been debated extensively with prior studies yielding conflicting results on patients' safety. Through the current body of literature, surgeons have not always been able to draw generalizable conclusions for all patient populations. Herein, we aim to provide a comprehensive, yet streamlined, overview of the common IS medications that surgeons are likely to encounter in practice in a wide variety of patients. Specifically, we report the risks of continuation versus temporary cessation of IS medications during elective surgery. The eight common IS medications investigated include: azathioprine, cyclosporine, hydroxychloroquine, infliximab, leflunomide, methotrexate, methylprednisolone, and mercaptopurine.

\section{Immunosuppression pharmacology}

Azathioprine inhibits DNA replication most usefully in T-lymphocytes, decreasing their overall function. It is used in renal transplant patients as an antirejection agent and is also used successfully in treating rheumatoid arthritis (RA). Azathioprine is metabolized in the liver by CYP450 to its metabolite mercaptopurine. It has a half-life elimination of two to five hours and is excreted in the urine and bile [1]

Cyclosporine is also used in transplant recipients as an antirejection agent, as well as for RA and psoriasis. Its mechanism of binding to cyclophilin inhibits calcineurin and subsequently decreases the dephosphorylation of nuclear factor of activated T-cells. This in turn decreases transcription and production of interleukin 2, thus downregulating the inflammatory process. The medication is metabolized hepatically by the CYP3A4 system and excreted mainly in the bile but also partially through the kidneys as well [2]. Cyclosporine has a half-life of 8 hours [3].

Hydroxychloroquine hinders the complement dependent reactions of antibodies and antigens and is used as second line therapy in those who have not responded well to previous RA, systemic lupus erythematosus (SLE), and malarial treatments. It has a half-life of about forty days, is metabolized in the liver and mostly excreted through urine [4].

Infliximab is given to adults for treatment of ankylosing spondylitis, Crohn's disease (CD), plaque psoriasis, psoriatic arthritis, RA, and ulcerative colitis (UC). As a chimeric monoclonal antibody, it decreases inflammation by binding to tumor necrosis factor alpha (TNFa). TNFa, when elevated, activates interleukins, eosinophils, and neutrophils, and improves migration of leukocytes [5]. The action of metabolism is unknown, though thought to be hepatically by CYP450. Infliximab has a half-life of 7-12 days [6]

Leflunomide is used to treat RA, juvenile idiopathic arthritis, cytomegalovirus, and also to prevent solid organ transplantation rejection. Leflunomide blocks pyrimidine synthesis to improve antiinflammatory effects by decreasing division of immune responsive cells. Its metabolism is hepatically and it has a mean half-life of eighteen to nineteen days [7].

Methotrexate binds to dihydrofolate reductase which inhibits dihydrofolate conversion to tetrahydrofolate. This inhibition decreases the activity of thymidylate synthetase and DNA production and thereby decreases lymphocyte proliferation. It has been used for treatment

Correspondence to: Michael R. Kammerer, Jefferson Bariatric and Metabolic Surgery, 211 S. 9th Street, Suite 402, Philadelphia, PA 19107, USA, Tel: (215) 955-0020; E-mail: Michael.Kammerer@jefferson.edu

Key words: immunosuppression, elective surgery

Received: March 05, 2017; Accepted: March 28, 2017; Published: March 30, 2017 
of many rheumatic and inflammatory diseases including ankylosing spondylitis, $\mathrm{CD}$, and psoriasis. It is also used as an adjunct chemotherapy agent in breast cancer, choriocarcinoma, osteosarcoma, non-hodgkin's lymphoma and acute lymphoblastic leukemia. Methotrexate is mostly metabolized in the liver by CYP450 but also in the gastrointestinal (GI) tract if taken orally. The half-life of methotrexate varies between 3 and 15 hours [8].

Methylprednisolone, being a corticosteroid, can be used to treat numerous inflammatory conditions including asthma, arthritis, SLE, chronic obstructive pulmonary disease (COPD) and UC. It helps to alter gene expression of proteins by binding to intracellular receptors. This binding in turn allows methylprednisolone to reduce inflammation by preventing cellular migration to the inflammatory site [9]. Metabolism of methylprednisolone is accomplished in the liver by CYP4503A4 and the metabolites are excreted renally. The oral half-life of methylprednisolone ranges from 1-4 hours [10].

Mercaptopurine is administered to patients with CD, UC, acute lymphoblastic leukemia, acute promyelocytic leukemia, and lymphoblastic lymphoma [11]. As an antimetabolite, it binds to DNA and RNA during synthesis and interrupts this process. Mercaptopurine is metabolized in the GI mucosa and in the liver by CYP450 producing active metabolites [12]. Mercaptopurine has a half-life of 1-1.5 hours and about fifty percent is excreted in the urine [11]. The other fifty percent remains as both inactive compounds and unknown compounds with unknown effects [12]

\section{Literature overview}

There is much overlap of the indications for, and the mechanisms of IS medications. Because of this, the literature discussion of risks of therapy and cessation is quite wide in breadth. The majority of evidence published on this topic seems to be in those undergoing solid organ transplantation, as these patients are the single largest cohort using IS medications continually. However, the remainder of evidence is in patients with rheumatic and inflammatory conditions undergoing orthopedic as well as other elective surgical procedures. Multiple studies exist which both support and oppose the use of IS in the perioperative period. Because there is no consensus between sources about when certain medications should be held or continued, the best approach is to individually analyze each medication and its own risks of maintenance or interruption perioperatively during an elective procedure. Therefore it helps to compare the risk of these two situations, published or theoretical, in order to systematically analyze each side of the question.

\section{Azathioprine}

A small-scale case series investigated seven pediatric solid organ transplant recipients (SOTRs) who continued use of IS medications during elective surgeries and found no increased risk for wound healing or infection [13]. More enlightening perhaps is a study of already immunosuppressed adults who have undergone solid organ transplantation. This particular study investigated 65 SOTRs who elected to have plastic and reconstructive surgeries. The data from 92 surgeries, of which some were emergent, showed there was no statistically significant increased risk of wound complication or infection associated with any IS medication [14]. The authors did state there is a high success rate for SOTRs undergoing elective and cosmetic surgeries using "careful" patient selection but did not describe what criteria contributes to this selection. They also concluded that urgent surgeries on SOTRs were $35 \%$ likely to have complications compared to the $6 \%$ chance during elective surgeries. Cases with complications had an average length of 168 minutes whereas uncomplicated cases averaged 70 minutes $(\mathrm{P}=0.03)$. A larger cohort repeating this study would yield more generalizable results [14].

Bongartz et al. [15] published a retrospective study of medical records on 462 adults at the Mayo Clinic Rochester with RA undergoing total hip and knee replacements which yielded no statistically significant correlation between infection risk after surgery and concurrent use of azathioprine and other IS medications [15]. A separate retrospective study looking at the medical records of 151 adults with UC undergoing colectomies while on azathioprine showed no relationship with rate of infection or complications; 12 out of 46 demonstrated early major complications but they were not attributed to azathioprine use. These complications included death, pelvic and urosepsis, abscess, reintervention of anastomosis, anastomotic leak, pneumonia, and small bowel obstruction. Moreover, this study showed that there was no significant correlation between complications and azathioprine use preoperatively $(\mathrm{P}=0.17)$ [16]. If Azathioprine is used perioperatively in adults with RA, renal function monitoring is recommended [17].

Azathioprine ultimately cannot be stopped in the context of transplant protection. However, the risk of stopping azathioprine in patients being treated for RA is less well understood. Concerns for a "flare-up" of RA has led many practitioners to encourage surgeons to continue therapy in the perioperative period. However, the risk of such a recurrence of symptoms versus the downsides of recurrence is not explicitly stated among the literature. In the absence of data, for most patients the risk of "flare-up" is likely not substantial.

\section{Cyclosporine}

There is a $3.5 \%$ increase in infection rates for adults with inflammatory bowel disease (IBD) undergoing surgery while on cyclosporine. However, the use of cyclosporine is still considered appropriate with special care postoperatively, which includes observations of deterioration in renal function and opportunistic infections [18].

Similar to Azathioprine, Cyclosporine therapy must be continued in SOTRs for protection of the transplanted organ, but cessation of treatment for RA and psoriasis has not been well studied. One retrospective study examined 46 publications evaluating the use of IS medications during procedures on adults with psoriasis, psoriatic arthritis, and IBD. These authors found that the continuation of cyclosporine during low-risk procedures on adults with psoriasis or psoriatic arthritis prevents "flare-ups" after procedures, though the exact percentage of prevention was not specified [3].

\section{Hydroxychloroquine}

The risks of continuation of hydroxychloroquine have varied in small-scale studies as the mechanism of action is not entirely understood [19]. Härle et al. [20] studied adults on hydroxychloroquine and antimalaria drugs and found no significant increase in infection or wound healing complications [20]. The toxicity profile of hydroxychloroquine contributes little risk in stopping or continuing use during surgery since individuals are able to recover quickly with this medication after outpatient operations [20,21]. It appears to be safe treatment for RA in the presence of elective surgery with renal function monitoring [17]. Hydroxychloroquine is not considered a potent immunosuppressant, and is therefore typically used perioperatively in orthopedic and other elective surgeries [21]. Thus there is no available evidence for risk of side effects resulting from stopping this medication abruptly. 


\section{Infliximab}

A study of 31 adults with RA who had foot and ankle surgery with continued use of infliximab and other TNFa inhibitors showed no increased risk of infection or wound healing complications $(\mathrm{P}=0.033)$. These adults were also typically on methotrexate and other disease modifying antirheumatic drugs [22].

A more generalizable study which analyzed 588 surgeries, from 26,932 patients, investigated usage of IS medications, including anti-TNF therapy, corticosteroids, and thiopurine, during elective colectomies in patients with UC. The data from the larger cohort of about 600 patients did not show a statistically significant correlation between IS medication use and postoperative complications [23].

Nevertheless, due to the enduring effects of infliximab, there is an increased risk of $13 \%$ and $43 \%$, respectively [24], in temporarily stopping therapy 2-5 half-lives before orthopaedic and abdominal surgery [20]. Thus if cessation of therapy is to be recommended, typical guidelines now advise stopping it 4-5 weeks before elective surgery as shorter temporary stoppage of infliximab increases the likelihood of psoriatic flare-up $(\mathrm{P}=0.003)$ [20].

\section{Leflunomide}

Bongartz et al. [15] examination of 462 patients with RA who underwent hip or knee replacements and were treated with methotrexate, azathioprine, or leflunomide yielded no statistically significant correlation between infection risk after surgery and concurrent use of IS medication [15]. Additionally, a European retrospective analysis of 42 patients, who underwent 77 procedures, with psoriasis or psoriatic arthritis investigated flare-ups and infections after elective skin, orthopedic, and cardiothoracic surgeries. Though the study is not generalizable due to small cohort size, the data did not show an increase in infection risk with continuation of IS medications during surgery [25].

Adults with RA or psoriatic arthritis undergoing orthopaedic surgery and taking leflunomide were studied in 2002-2003. The authors found that those on leflunomide at time of surgery had a statistically significant increased risk of infection during wound healing $(\mathrm{P}=0.01)$ [26]. With the conflicting numbers regarding wound healing complications, their recommendation was to wash out leflunomide using cholestyramine in adults with RA 1 week before surgery $[17,20]$. The wash out phase ensures there is no drug left in the system [20].

Härle et al. [20] also showed a $6.1 \%$ infection rate in adults with RA who continued their therapy and a $6.3 \%$ infection rate in those who temporarily stopped their leflunomide. However, the number of patients in the study was relatively small and the results were nonsignificant. A comprehensive overview of continuation, temporary stoppage, and a complete wash out of leflunomide in patients before receiving elective surgery has yet to be investigated [20].

\section{Methotrexate}

Härle et al. [20] retrospective review of 47 previously published studies recommended stopping methotrexate one week before complex surgery and resuming treatment one week after surgery, if subjects have no history of wound healing problems. This result was shown in a small cohort where a 7 -day pause of treatment yielded no postoperative infection whereas $25 \%$ of those continuing treatment developed infection [20]. Conversely a separate retrospective review of postoperative outcomes of 172 adults on methotrexate for RA undergoing elective orthopedic surgery showed continuation of methotrexate during surgery had a lower rate of infection than temporarily stopping its use $(\mathrm{P}=0.0045)$. Moreover, the group who continued methotrexate had a lower rate of infection than those who had no historical use of the drug $(\mathrm{P}=0.038)$ [19].

As methotrexate regulates immune responses instead of inhibiting these responses, most studies have shown no significant increase in wound healing complications or infection rate with continuation during elective surgeries [20]. For adults with RA undergoing wrist and hand surgeries while taking methotrexate, there is a $3.3 \%$ rate of wound infection which increases to $33 \%$ for those with diabetes [27]. Methotrexate appears to have little risk of infection in adults with RA and thus its use is recommended perioperatively with renal function monitoring [17].

\section{Methylprednisolone}

Corticosteroids are typically assumed to significantly increase the risk of infection or surgical complication after elective surgery. However, a prospective randomized analysis demonstrated limited clinical evidence on the benefits and risks of using corticosteroids perioperatively [28].

On the contrary, corticosteroid use preoperatively is a risk factor for infection in RA patients as shown in a literature review of 74 studies. If methylprednisolone is continued during surgery, patients require heightened monitoring for infectious complications postoperatively [29].

\section{Mercaptopurine}

Kumar et al. [18] literature analysis consisting of 84 articles regarding perioperative management of patients with IBD concluded that continued use of mercaptopurine does not negatively influence surgical outcomes or increase morbidity following elective surgery. There was no increase in post-operative infection rates for adults with IBD undergoing bowel procedures while on glucocorticoids when compared to adults on purine analogues [18]. Thus, continued use of mercaptopurine likely does not negatively impact post-operative complication rates for adults with IBD.

Treatment with mercaptopurine, an antimetabolite, should temporarily stop on the same day as surgery in adults with IBD as the drug may contribute to bone marrow suppression, hepatitis, leukopenia, and pancreatitis. Kumar et al. [18] suggested that mercaptopurine can be readministered within the first 3 days following surgery contingent upon normal renal functions [18].

\section{Conclusion}

It is best to have a comprehensive medical armamentarium to lower the risk of perioperative complication; however this task proves difficult with contradicting findings in the literature regarding the effects of IS use during surgeries. Moreover, this difficulty includes the implications of IS medication withdrawal, its potential to interrupt the disease therapy, and its potential anesthetic agent interference [30]. Current literature does not overwhelmingly support the claim that the use of immunomodulators perioperatively increase the infection rate or wound healing complication rate despite the majority of rheumatologists and/or surgeons preferring to withhold IS medications. It seems best for physicians to assess the use of immunomodulators on a case-by-case basis [30].

The risk of complications following elective surgical procedures needs to be contrasted against the risk of stopping IS medication use. 
This comparison is likely different for each procedure and medication in question. Higher risk surgical procedures, usually have higher rewards such as is the case in oncologic resections. In these cases, the small risk of complication due to IS medication use may be deemed acceptable, however in the case of elective surgical procedures, which usually carry a smaller overall surgical risk, the additional risk of complications due to IS medication use may not be an acceptable risk. The literature seems to indicate that adults can stay on azathioprine, cyclosporine, hydroxychloroquine, infliximab, and methotrexate, with only theoretical but not data-driven increased risk. However, cessation of leflunomide, mercaptopurine, and methylprednisolone seems to be recommended as these IS medications appear to have a theoretical risk that outweighs any benefit.

Overall, data on the use of IS medications during elective surgeries either demands for repeated investigation for more generalizable results or has no statistically significant results regarding the rate of post-surgical complications. In the latter situation the standard complication rate of the surgical procedure and its perceived benefits should be taken into account when deciding if IS medication would be contributing a significant additional risk, and therefore be held. The trend does appear to show that elective surgery is manageable for selected patients on IS medications given the advancements in surgical procedures and IS therapy.

\section{Disclosure}

The authors declared no conflict of interest.

\section{Funding}

There was no outside funding for this project.

\section{References}

1. Azathrioprine (2017) Drug Information. In: UpToDate, UpToDate, Waltham, MA. (Accessed on February 16, 2017)

2. Cyclosporine (2017) Drug Information. In: UpToDate, UpToDate, Waltham, MA. (Accessed on February 16, 2017)

3. Choi YM, Debbaneh M, Weinberg JM, Yamauchi PS, Van Voorhees AS, et al. (2016) From the Medical Board of the National Psoriasis Foundation: Perioperative management of systemic immunomodulatory agents in patients with psoriasis and psoriatic arthritis. J Amer Acad Dermatol 75: 798-805.

4. Hydroxychloroquine (2017) Drug Information. In: UpToDate, UpToDate, Waltham, MA. (Accessed on February 16, 2017)

5. Infliximab (including biosimilars of infliximab) (2017) Drug information. In: UpToDate, UpToDate, Waltham, MA. (Accessed on February 16, 2017)

6. Remicade (2017) In: Epocrates, Epocrates, San Mateo, CA. (Accessed on February, $16,2017)$

7. Leflunomide (2017) Drug information. In: UpToDate, UpToDate, Waltham, MA. (Accessed on February 16, 2017)

8. Methotrexate (2017) Drug information. In: UpToDate, UpToDate, Waltham, MA. (Accessed on February 16, 2017)

9. Methylprednisolone (2017) Drug Information. In: UpToDate, UpToDate, Waltham, MA. (Accessed on February 16, 2017)
10. Methylprednisolone (2017) In: Epocrates, Epocrates, San Mateo, CA. (Accessed on February, 16, 2017)

11. Mercaptopurine (2017) Drug Information. In: UpToDate, UpToDate, Waltham, MA (Accessed on February 16, 2017)

12. Mercaptopurine (2017) In: Epocrates, Epocrates, San Mateo, CA. (Accessed on February, 16, 2017)

13. Ayub B, Young NM (2016) Cochlear implantation of solid organ transplant patients receiving immunosuppressive therapy. Int J Pediatric Otorhinolaryngol 91: 19-22.

14. Zellner E, Lentz R, Chuang C, Steinbacher D (2016) Complications Following Plastic Surgery in Solid Organ Transplant Recipients: A Descriptive Cohort Study. J Aesthetic $\&$ Reconstructive Surgery.

15. Bongartz T, Halligan CS, Osmon DR, Reinalda MS, Bamlet WR, et al. (2008) Incidence and risk factors of prosthetic joint infection after total hip or knee replacement in patients with rheumatoid arthritis. Arthritis Rheum 59: 1713-1720. [Crossref]

16. Mahadevan U, Loftus EV, Tremaine WJ, Pemberton JH, Harmsen WS, et al. (2002) Azathioprine or 6-mercaptopurine before colectomy for ulcerative colitis is no associated with increased postoperative complications. Inflam Bowel Dis 8: 311-316.

17. Goodman SM (2015) Rheumatoid arthritis: perioperative management of biologics and DMARDs. Semin Arthritis Rheum 44: 627-632. [Crossref]

18. Kumar A, Auron M, Aneja A, Mohr F, Jain A, et al. (2011) Inflammatory Bowe Disease: Perioperative Pharmacological Considerations. Mayo Clinic Proceedings 86 748-757.

19. Agarwal S, Das A (2016) Postoperative Complications For Patients With Rheumatoid Arthritis Undergoing Elective Orthopedic Procedures With And Without Methotrexate. J Adv Med Dent Sci Res 4: 28.

20. Härle P, Straub RH, Fleck M (2010) Perioperative management of immunosuppression in rheumatic diseases - what to do?. Rheumatology International 30: 999-1004.

21. Scanzello CR, Figgie MP, Nestor BJ, Goodman SM (2006) Perioperative management of medications used in the treatment of rheumatoid arthritis. HSS $J$ 2: 141-147. [Crossref]

22. Bibbo C, Goldberg JW (2004) Infectious and healing complications after elective orthopaedic foot and ankle surgery during tumor necrosis factor-alpha inhibition therapy. Foot Ankle Int 25: 331-335. [Crossref]

23. Bewtra M, Newcomb C, Wu Q, Lewis JD (2013) 1007 Immunosuppressant Therapy Prior to Elective Surgery Is Not Associated With Increased Post-Operative Morbidity in Ulcerative Colitis. Gastroenterology 144: S-188.

24. Ruyssen-Witrand A, Gossec L, Salliot C, Luc M, Duclos M, et al. (2007) Complication rates of 127 surgical procedures performed in rheumatic patients receiving tumor necrosis factor alpha blockers. Clin Exp Rheumatol 25: 430-436. [Crossref]

25. Bakkour W, Purssell H, Chinoy H, Griffiths CE, Warren RB (2016) The risk of postoperative complications in psoriasis and psoriatic arthritis patients on biologic therapy undergoing surgical procedures. J Eur Acad Dermatol Venereol 30: 86-91. [Crossref]

26. Fuerst M, Möhl H, Baumgärtel K, Rüther W (2006) Leflunomide increases the risk of early healing complications in patients with rheumatoid arthritis undergoing elective orthopedic surgery. Rheumatol Int 26: 1138-1142. [Crossref]

27. Jain A, Witbreuk M, Ball C, Nanchahal J (2002) Influence of steroids and methotrexate on wound complications after elective rheumatoid hand and wrist surgery. $J$ Hand Surg 27: 449-455.

28. Grennan DM, Gray J, Loudon J, Fear S (2001) Methotrexate and early postoperative complications in patients with rheumatoid arthritis undergoing elective orthopaedic surgery. Annals Rheum Dis 60: 214-217.

29. Krause ML, Matteson EL (2014) Perioperative management of the patient with rheumatoid arthritis. World J Orthop 5: 283-291. [Crossref]

30. Whinney C (2009) Perioperative medication management: general principles and practical applications. Cleve Clin J Med 76: S126-S132. [Crossref]

Copyright: (C2017 Koons K. This is an open-access article distributed under the terms of the Creative Commons Attribution License, which permits unrestricted use, distribution, and reproduction in any medium, provided the original author and source are credited. 